\title{
The Effects to Trend the Suitable OS Platform
}

\author{
Aso M. Aladdin ${ }^{1}$, Yad N. Bakir², Sarwar I. Saeed ${ }^{3}$ \\ 1,2,3 Charmo University, College of Education and Natural Science, Peshawa Street,Chamchamal/Sulaimani, \\ University of Human Development, College of Science and Technology, Goizha Mountain, Sulaimani, Iraq. \\ ${ }^{1}$ Iraq,aso.aladdin@charmouniversity.org, aso.aladdin@uhd.edu.iq, 2Iraq, \\ yad.bakir@charmouniversity.org,yad.bakir@uhd.edu.iq, 3Iraq, sarwar.saeed@charmouniversity.org
}

\begin{abstract}
Technically, software is a part of electronic devices that is responsible for managing hardware. The signaling communication between hardware and software ultimately controls electronic devices, and is known as Operating System (OS). For the purpose of selecting the best platform for clients, users should study functionalities, securities, graphic interfaces and usability of the different OS platforms. Therefore, this research focuses on choosing the suitable OS platform for user in academic and non-academic environments, according to demanding users' companionability. It also explains the first type of OS which can be utilized openly. In addition to that, it concentrates on some general significant aspects that are useful for OS users.
\end{abstract}

Keywords: Operating System (OS), Windows, Macintosh, Ubuntu, Functionality, Security, Applications, Affects

Subject Classification: Computer Science and Information Technology- Computer System - Operating System

Type (Method/Approach): Historical Inquiry; Literary Analysis; Survey

Language: English

Date of Publication: 2018-07-30

DOI: $10.24297 /$ jns.v5i1.7528

ISSN: 2393-9257

Volume: 05 Issue: 01

Journal: Journal of Advances in Natural Sciences

Website: https://cirworld.com

This work is licensed under a Creative Commons Attribution 4.0 International License. 


\section{INTRODUCTION}

This paper sheds light on answering the common question on how computer works and how users interact with it. Technically, software is responsible for managing hardware which is known as an Operating System (OS). OS controls the distribution of resources and services, for example memory, processors, devices and information. Therefore, we will focus on OS overview.

Thus, OS is intermediate software between users and computer hardware. With OS, Users can execute programs usefully, conveniently, powerfully and efficiently [1]. Hence, according to Silberschatz, Galvin and Gagne (2003) an OS "is a program that acts as an interface between users and the computer hardware, it also controls the execution of all kinds of programs." [2] Look at figure 1.

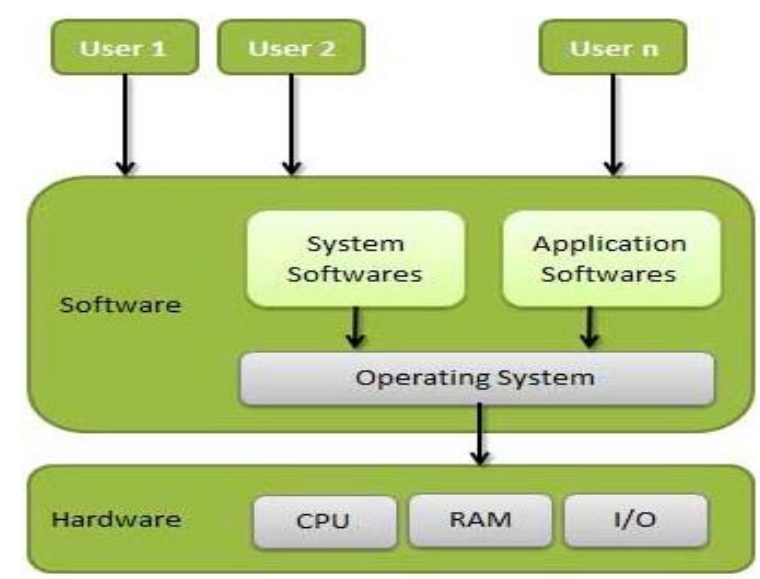

Figure 1: Abstract view for Computer System

To consider different types OS, the emphasis should be on some important functions that point out and differentiate the effort of the OS, according to the users and daily exercising Operation. Therefore, these functions may affect the ratio of the users and some programmers for selecting the best type of an OS. Besides, these are normally organized into directories in order to provide easy navigation and usage [3]. The above mentioned functions and activates are more crucial to determine the best OS for users, such as security and ensuring that unauthorized user do not have access to the system, control over system performance, job accounting, error detecting aids and coordination between other software and users [1]. Furthermore, OS has a user interface or GUI which accepts requests from users and interprets them to the rest of the operating system. It also helps the users to choose the best simple design of OS [4].

\section{OS CLASSIFICATION}

OS classified according to numbers of users and tasks during operation [5].

There are two types of OS depending on the number of user:

- Single User OS that allows only one user to work on it at a time. Example of Single User OS is DOS (Disk Operating System).

- Multi User OS which allows more than one user to work on it simultaneously, examples of multi user OS is Windows 7, Windows Vista, Windows-XP etc.

Based on the number of tasks O.S, categorized into two types:

- Single-Tasking OS that supports only one task at a time, for instance, DOS OS type.

- Multi-Tasking OS that supports more than one task at a time, for instance, Windows 7, Windows-XP etc. 


\section{OS MODELS}

Most people use the OS that comes with their computer, but it is possible to upgrade or even change it and update it the for organizational purpose. There are many various Models of OS. Methodically, each does the same thing, they control all input, processing and output, but deferent from design and usability.

I. DOS (Disk Operating System): one of the first OS for the personal computer. When users turn the computer on, they could see the command prompt, which appeares like $c: 1>$ wplwp.exe. It also is known as a command-line interface (CMD). Nowadays, another word it is not "user friendly" by normal users [6].

II. Windows: The Windows OS is a product of Microsoft organization and a GUI (graphical user interface) OS. The most popular ones are Windows 10 (2015) Windows 8 (2012) Windows 7 (2009), Windows Vista (2007), and Windows XP (2001). Also, "user friendly" familiarizes this operating system and is said to have WIMP features (Windows, Icons, Menus, Pointing device) [7].

III. Macintosh (Mac OS): It is a product of Apple organization. Suitably, has its own OS with a GUI and WIMP features.

IV. UNIX - Linux Ubintu (The PC version of UNIX): originally, UNIX and Linux were generated with a command-line interface, but recently have added GUI enhancements.

User-friendly is a term used for software system if it is easier to use for users and created by GUI. The current GUI interfaces provided by Windows and Mac operating systems that are more familiar to use than other previous systems [8].

\section{OS USER}

It is important to have knowledge of various types of OS and their effects on multiple numbers of users. Each type of OS has advantages and disadvantages rendering the user's reliability and requirements. It is not straight forward to determine the exact percentage users. As a result, it is almost impossible to obtain accurate numbers for OS market share, due to the nature of how share market is traditionally calculated. Conventionally, most market share charts are depended on around sales instead of depending on stats from online activity or user polls.
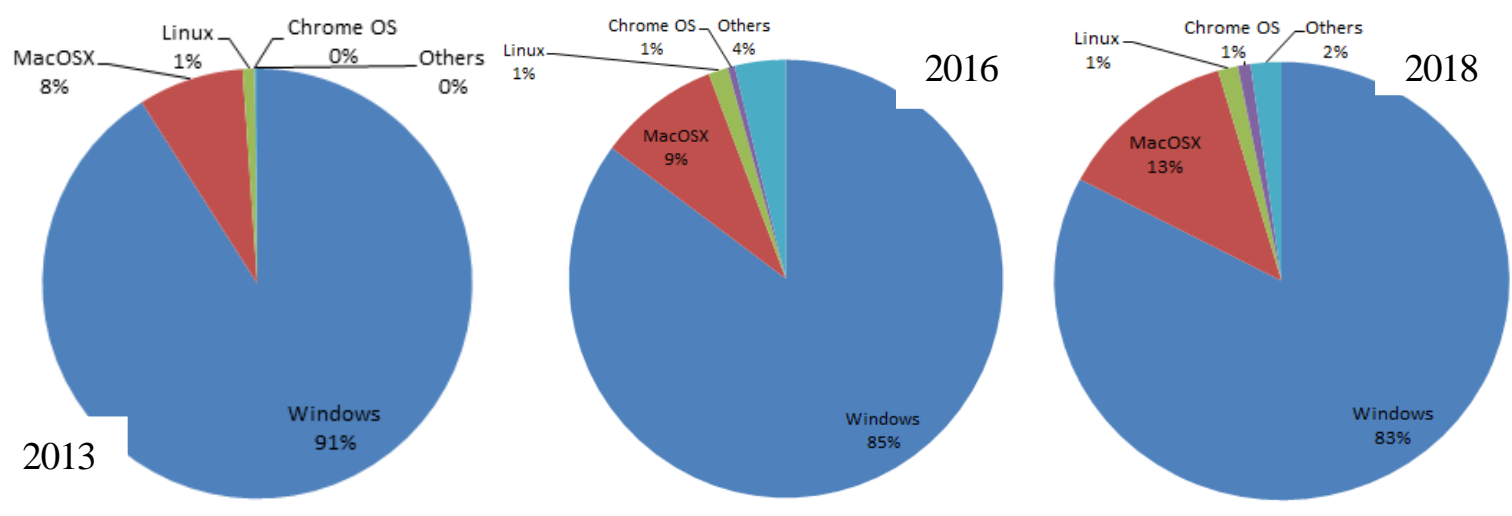

Figure 2: Compare Computer OS users' percentage between 2013, 2016 and 2018

(The Statistics Portal, 2018) 
Consequently, all the statistics that depend on sales chart and market share, recommend windows as a first OS, which are sold in the world or specific area. According to Market Share Statistics for Internet Technologies (2016) the desktop share OS that depends on the sale of OS in the world on 2013, 2016 and 2018, windows are used widely and Mac OS comes as in the second place after that Linux [9]. Look at Figure 2.

Analyzers look at the statistic of using OS by version; however it generates a few surprises. XP continues to slide; Windows 8 and 10 is very slowly gaining attraction. However, window 8 and 10 is a new version Windows and Microsoft still controls usage in the declining market for traditional PCs. Despite the fact that statistic shows a few unexpected trends.

On the other hand, being less effective interface, lack of innovation to the desktop interface, developers' abhorrence, as well as tablet, smartphone, and desktop competition, are some other factors that caused Windows 8 failure. Another reason can be traced back to the feasibility of Windows 7, that made impossible for users to consider another alternative. Therefore; Microsoft has designed and developed Windows 10 [10].
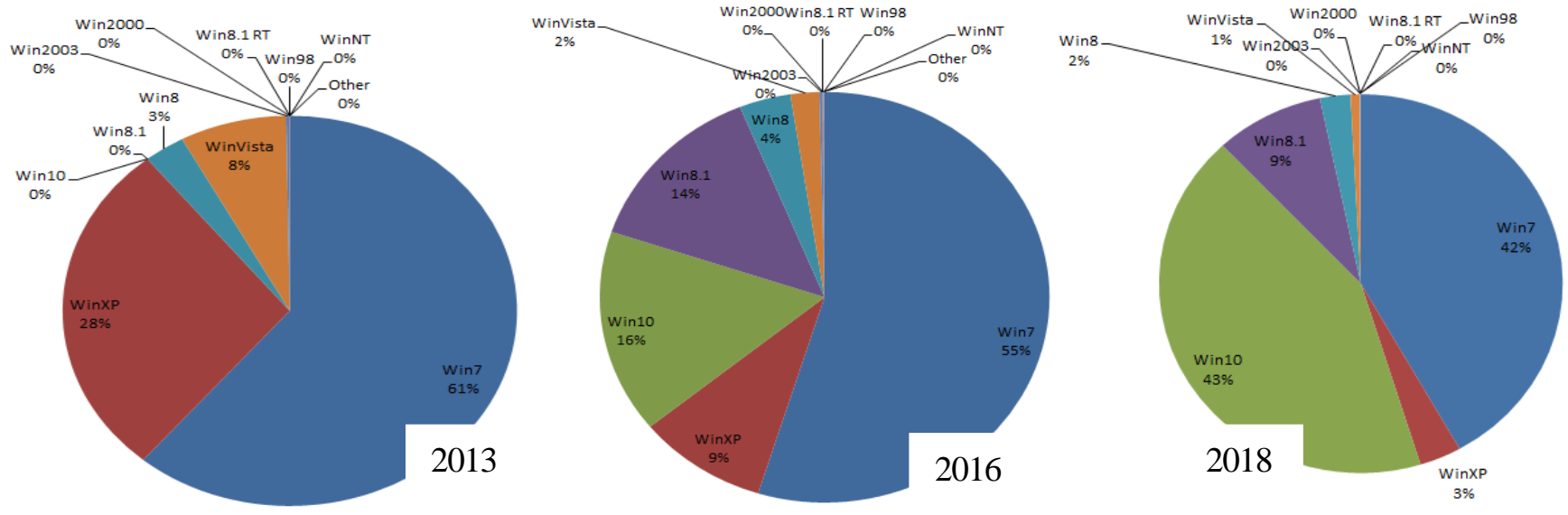

Figure 3: The impact of market sharing on OS User for Computer Devices (The Statistics Portal, 2018)

Looking at the comparison between 201, 2015 and 2018; changed from 46.64 percent to 52.34 percent. Unlike, Windows XP is still dropping [9]. Nevertheless, the predictions show that Wind 7 and Win 10 should be given great user and shares, but Windows 8 failure is actually greater than it appears as shown in the previous data in Figure 3. Besides, according to the data users are not familiar with Windows 10 at first but in time they will be familiar and flexible with it. Look at Figure 4 [11].
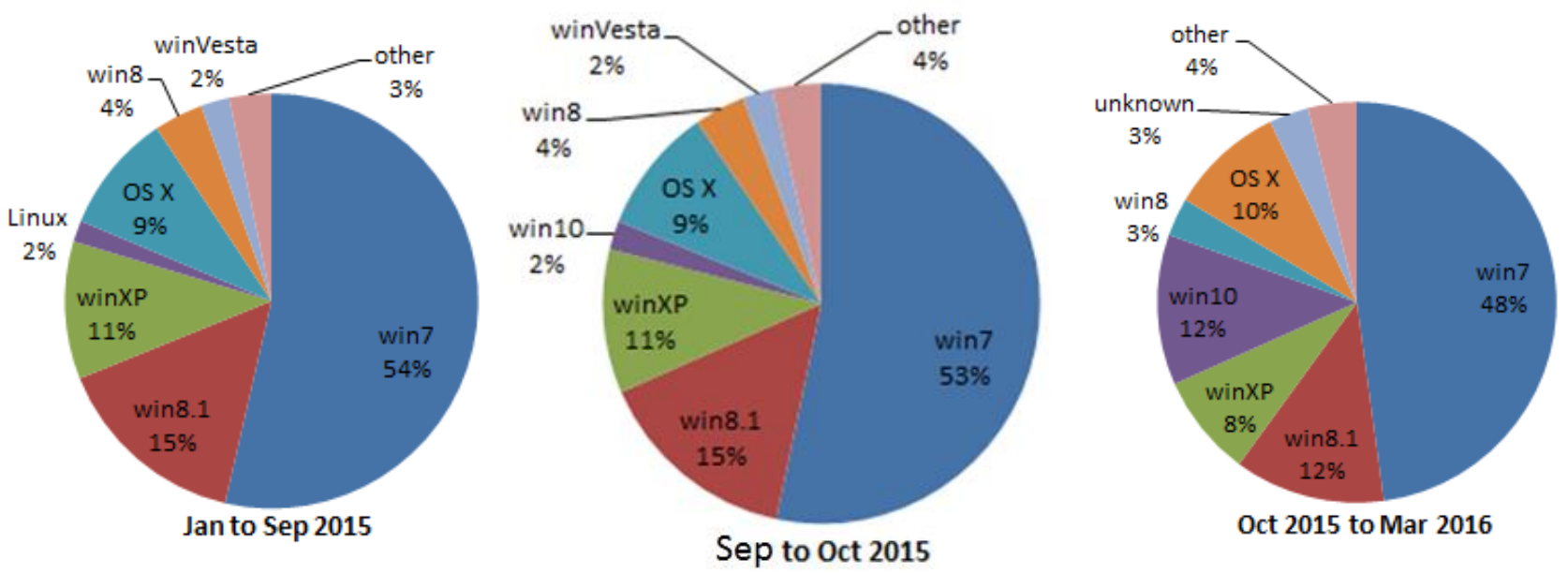

Figure 4: Static counter from Desktop users for top 7 OS (StatCounter, 2018) 
This statistic shows that OS users' for PC have changed according to the OS developers (organization), usability, user's education, interface, liability, security and the cost of applications for users.

\section{OS COUNTENANCE}

Currently there are two common operating systems in the world which are hugely used and shared by normal users. Linux or Ubuntu are also another OSs that are used by developers, based on the advantage and disadvantage like, user interface, compatibility, technical support, viruses (Security), speed and price. This section is dedicated to the unbiased comparison between Windows, Macintosh and Linux.

\subsection{Windows}

Windows is the most popular OS in the world, with almost $90 \%$ of the OS market share. It is also utilized in commercial buildings, industrial facilities, as well as home computers. Generally, first windows have been introduced in 1985 [known as Windows 1.0 which included MS-DOS file management], is a very developed and completed software system [12].

Pros according to (Compatibility, Technical support, Functionality, Cost)

- Most of the applications, drivers or games will run on Windows; therefore, it is compatible.

- Number of Technical staffs and personnel who are experienced and able to provide after care support is enormous.

- It has multiple diverse functions. Thus, user can find out several functions that are used easier than those of the other operating systems [13].

- It is sold for a reasonable price and users can have cost effective user licenses and application [14].

Cons (depend on Security)

Due to the fact that Malware might easily attack windows, users need to buy activated antivirus program. These threats and malwares affect the windows and make running slower [13].

\subsection{Macintosh}

Macintosh is also a big player in the field. Indeed, Apple's Macintosh OS is older than windows but it has fewer users than the latter. Moreover, it is the first successful graphical-based OS, which means progressing by GUI.

Pros according to (Security, reliability, design)

- Apple Macs get almost no viruses and due to window's superior market share.

- Mac is reliable because it only runs on Apple computers.

- Mac User Interface is better than windows and other OSs because of splendid design [15].

Cons (depend on Cost \& Application)

The problem cannot be effortlessly tackled. Apple hardware is expensive and Mac costs even more than Windows. Users already have a computer and cannot install MAC OS on it unless it's an Apple Hardware unless they download boot camps. Subsequently, user must buy an Apple device.

The second drawback of Mac OS X is showed that most of the applications and games are not free. Users have to buy them from the Apple store [16]. 


\subsection{Linux}

It is smaller but can be effective and developed. Linux is Genuinely Not UNIX (GNU) and GNU is an OS that merely uses free software and depend on the General Public License. Hence, it means Linux is FREE; user is capable of downloading, modifying, upgrading and rearranging it without spending a dime!

Pros according to (Cost, Functionality, Security)

- Linux is free to be downloaded and is able to be installed and modified on each computer device without purchasing it [14].

- Linux is not a polished OS and it includes a kernel. For using kernel needs extra software in order to be bundled with Linux. Several of these bundles exist (this called "distributions" or simply "distros"). The most popular ones include Ubuntu, Mint and Fedora [17].

- Linux is more vulnerable to viruses because it is open source, that's why; Linux is still vulnerable to a large number of viruses [18].

Cons (depend on Usability, Application)

Some "distros" are using easily, but most of them should have decent computer knowledge to start using. The same is true for Mac, it obtains only a few percent of the market share; likewise, it does not have a great numbers of programs and games as Windows [18].

\section{INVESTIGATING THE OS EFFECT ON USERS}

This paper explains the usability of the best OS platform for academic users in the educational domain and also non-academic users. It also helps them in cherry picking the best OS system according to the demand of their professional environments. Questioners and IT demands of a number of centers and company were analyzed to reach a conclusion.. The investigation includes tackling some crucial topics, such us, OS usability, security, functionality, application's compatibilities and etc. The data have collected from Universities and Institutes in North of Iraq "Kurdistan Region" and Company officials in that Region.

$\% 100$ of participants have used and focused on different versions of Windows as researchers has expected. Most of students and teachers exaggerate that the smart devices are running by Windows on the laboratory PC and himself/herself computer. Subsequently, most of company are comfortable with different Windows especially Win7. On the other hand, some lecturers from different departments illustrated the some applications just run on Macintosh OS such as TV programs and development of new applications. Therefore, the ratio of Macintosh OS usage is arrived near \%42 as shown in figure 5. Besides, a few of users are used Ubuntu (Linux and Unix OS) because they are studied from the college of sciences and obliged to be used for data analyzing and designing applications and websites especially a lot of clients used for analyzing in bioinformatics science, look at Table 2. In contrary, the staffs of the colleges of humanities except Media departments have not any idea about Macintosh OS and Ubuntu.

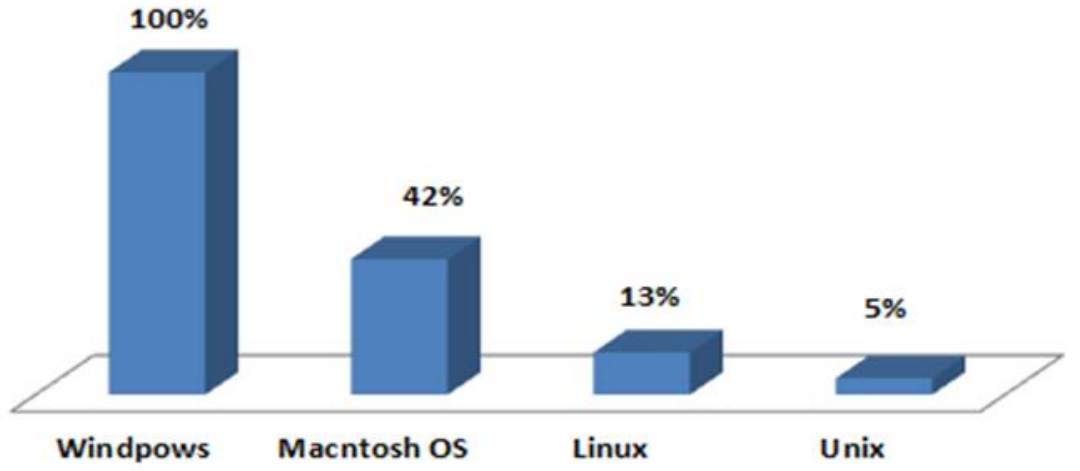

Figure 5: Percentage OS users for electronic devices (Computer) according to the Kurdistan Region Sample 


\subsection{Windows Functionalities and Affects}

The statistics in the previous sections indicate that most proportion of users especially academic staffs depend on the Windows OS. It is obvious that Windows have various types which have developed by Microsoft Company according to time and efficiency.

Each type has special functionalities and has different effects on consumers. Thus, these causes make dissimilar users ratio. When look at figure 6 , the percentage of Windows 7 usage is highest number of users and demand. According to the statistics which collected from the teachers and the students depending upon his/her computers and laboratory computers, Windows7 is dedicated for them. While windows 8 is less demanded than Windows7; however, Windows 8 is newer than the other. Besides, Windows 10 is the most updated version of windows designed by Microsoft Corporation Company. Strangely, The Company has decided to use and depend on Windows Vista because of the lack of users and unsuccessful functionality.

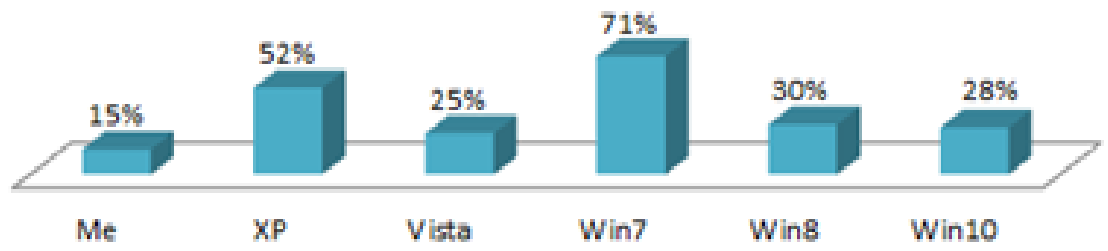

Figure 6: the percentage Windows users

Nevertheless, the data collected from the academic scholars, who were both equally normal users and $\Pi$ professionals, it determines that the users absorb windows7 because easier to use conferring to the best design and companionable with applications and games. Likewise, the users showed preference to Win7, XP and Win10 for resolving network problems and detecting or remediating errors quickly. Outlandishly, maximum of participants have not had ideas about the price for original application because of depending on those apps and games that are available for free, users or download them from free websites.

A lot of Windows push their security for the viruses and spyware to the min (minimum?). correspondingly, largest ratio of participants believe that all windows has the same secure defender for the viruses and malwares. As a result, it is better that users depend on the Anti-Viruses during downloading Windows OS or Android OS on the hardware device.

Table 1: The differences of functionality in Windows by percentage

\begin{tabular}{|c|c|c|c|c|c|c|c|c|}
\hline Functionalities & $\mathrm{Me}$ & $X P$ & Vista & 7 & 8 & 10 & Similar & No idea \\
\hline $\begin{array}{l}\text { Easy to use and efficient design } \\
\text { interface }\end{array}$ & $\% 5$ & $\% 16$ & $\% 4$ & $\% 48$ & $\% 10$ & $\% 13$ & $\% 2$ & $\% 2$ \\
\hline $\begin{array}{l}\text { The largest library of programs, } \\
\text { applications and games for other } \\
\text { languages }\end{array}$ & $\% 2$ & $\% 9$ & $\% 2$ & $\% 32$ & $\% 11$ & $\% 22$ & $\% 4$ & $\% 18$ \\
\hline $\begin{array}{l}\text { More compatible for most of the } \\
\text { programs, applications and games }\end{array}$ & $\% 2$ & $\% 11$ & $\% 1$ & $\% 44$ & $\% 9$ & $\% 20$ & $\% 3$ & $\% 10$ \\
\hline $\begin{array}{l}\text { Normal price for original applications } \\
\text { and games }\end{array}$ & $\% 1$ & $\% 12$ & $\% 3$ & $\% 25$ & $\% 6$ & $\% 18$ & $\% 6$ & $\% 29$ \\
\hline Easier to fix networking problem & $\% 3$ & $\% 24$ & $\% 2$ & $\% 36$ & $\% 10$ & $\% 11$ & $\% 2$ & $\% 12$ \\
\hline $\begin{array}{l}\text { Easier maintenance to avoid system } \\
\text { errors and enhancing performance }\end{array}$ & $\% 1$ & $\% 21$ & $\% 3$ & $\% 36$ & $\% 7$ & $\% 14$ & $\% 5$ & $\% 13$ \\
\hline $\begin{array}{l}\text { Security system from viruses, spyware, } \\
\text { and adware }\end{array}$ & $4 \%$ & $9 \%$ & $4 \%$ & $18 \%$ & $5 \%$ & $16 \%$ & $24 \%$ & $20 \%$ \\
\hline
\end{tabular}




\subsection{Mac OS Affects}

Obtaining original programs, applications or games for Mac OS X is developed and most of them are paid version, as a result, this type of OS is relatively more expensive. According to the Figure 7, most of clients have the same opinions about Mac OS, because Mac OS original apps and game is more expensive than other types of OS. Also, Most of none- academics doesn't depend on Mac OS because it is not straight forward to be learned beginners face more difficulty especially in designing according to Graphical User Interface. However, Mac OS is more secure and reliable. Look at Table 2.

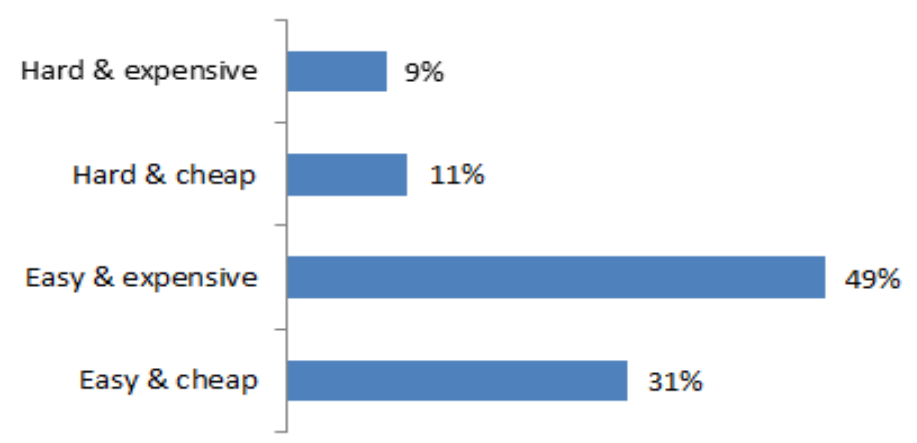

Figure 7: Percentage of Mac OS Users affects

\subsection{Ubuntu Affects}

It is worth observing that \% 18 of the participant used Ubuntu who has developed the programing language and have a postgraduate degree in science. As shown at Figure 8; more than fifty percent of clients who utilized the type of this OS, found it easy for learning and beneficial for data analysis. It also states that users in the scientific laboratory during working, in the chemical and bioinformatics laboratory massively depend on it. Nonetheless, most of consumers do not have knowledge about functionalities as shown in the table 2 .

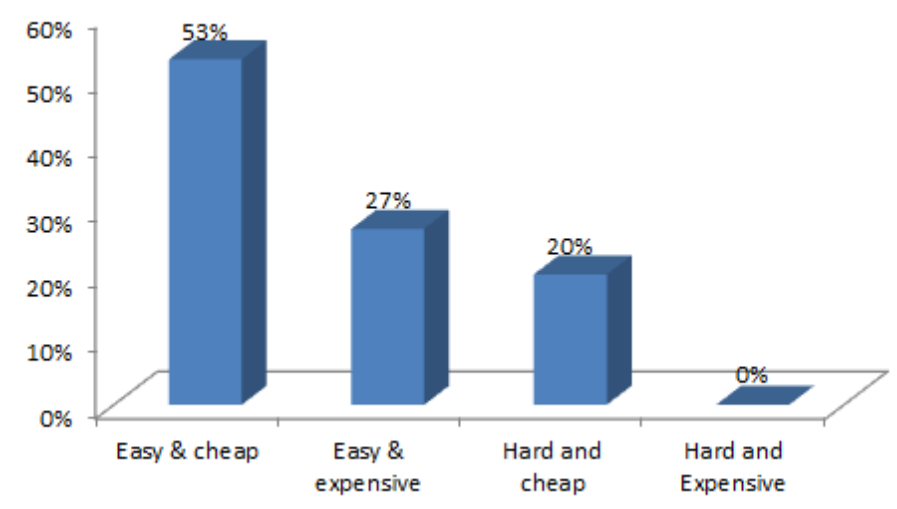

Figure 8: Percentage of Ubuntu OS Users affects 
Table 2: Users functionalities agreement for different types of OS

\begin{tabular}{|cccc}
\hline functions & Windows & Mac OS X & Ubuntu \\
\hline Customizable \& Compatible & $80 \%$ & $17 \%$ & $3 \%$ \\
\hline Largest Different Languages Library & $66 \%$ & $29 \%$ & $5 \%$ \\
\hline Free Applications & $73 \%$ & $18 \%$ & $9 \%$ \\
\hline Network Support & $74 \%$ & $16 \%$ & $10 \%$ \\
\hline Technical Help & $65 \%$ & $30 \%$ & $5 \%$ \\
\hline Security and Reliability & $38 \%$ & $51 \%$ & $11 \%$ \\
\hline
\end{tabular}

\section{CONCLUSION AND SUMMARY}

With the fast pace of technological advancement; the demands of using electronic devices with best facilities will increase dramatically. Due to this fact, this paper discussed evaluating the best operating system for electronic devices depending on functionalities, securities, graphic interfaces and usability of the different OS platforms. This research relies on both static OS selling charts and data gained from questioners held inside universities and companies. As a result of first technique, sets of data have been collected in 2013, 2015 and 2018, which shows that a majority of users bought windows OS.

To some extent, Windows 7 is at the top OS usability, according to users recommendations, Windows 8 and 10 placed in second and third position respectively. The data of questioners have displayed that nearly all participants chose Windows OS, with few users of Linux and Macintosh.

To conclude, Windows OS almost enforced their presence all over the world due to having a huge number of advantages over other OS, instance of good points compatibility, un-limiting users and economically. Mac has a significant role after windows as it secures reliability, splendid design and almost no viruses. The reason why Linux has the smallest number of users is the free downloading programs, demanding kernels an adjacent part, and vulnerability to viruses. Finally, it has been inferred that the best platform of OS based on higher functionalities percentages and advantages among the targeted users is windows. Approximately all users use Windows then Macintosh and Ubuntu OS respectively.

\section{REFERENCE}

[1] Tanenbaum, S. A. \& Bos, H. (2014) Modern Operating Systems. 4th Ed. New Jersey: Pearson.

[2] Silberschatz, A., Galvin P. B. \& Gagne, G. (2008) Operating System Concepts. 8th ed. USA: John Wiley \& Sons.

[3] Robert Milton Underwood, Jr. (2000) Device Drivers: Their Function in an Operating System [online] Available at: http://www.homesaustin.com/Documents/DeviceDrivers.pdf [Accessed 3 March 2016]

[4] Feske, N. \& Helmuth, C. (2007) Design of the Bastei OS Architecture [online] Available at: http://www.qucosa.de/fileadmin/data/qucosa/ [Accessed 2 March 2018]

[5] Burgess, M. (2001) A Short Introduction to Operating Systems [online] Available at: http://markburgess.org/os/os.pdf [Accessed 3 March 2018] 
[6] Swayne, D. (2003) Microsoft's Disk Operating System [online] Available at: https://users.cs.jmu.edu/ [Accessed 28 February 2018]

[7] Nirmal R. K. and Mishra N. (2011) 3D Graphical User Interface on personal computer using p5 Data Glove. International Journal of Computer Science Issues, Vol. 8 (5), No.1: pp. 155-160

[8] Petrie, H. and Bevan, N. (2009) the evaluation of accessibility, usability and user experience. The Universal Access Handbook, C Stepanidis (ed), CRC Press, [online] Available at: www.crcpress.com/product/isbn/9780805862805 [Accessed 29 February 2018]

[9] Market Share Statistics for Internet Technologies (2016) Desktop Operating System Market Share [online] Available at: https://www.netmarketshare.com/ [Accessed 14 March 2016]

[10] Vaughan-Nichols S. J. (2013) Five Reasons why Windows 8 has failed [online] Available at: http://www.zdnet.com/article/ [Accessed 6 March 2018]

[11] StatCounter (2016) Find the most popular operating systems [online] Available at: http://gs.statcounter.com/ [Accessed 14 March 2018]

[12] DuCharme, B. (2001) the Operating System Handbook: Fake Your Way through Minis and Mainframes [online] Available at: http://www.snee.com/bob/opsys/part4os400.pdf [Accessed 10 March 2018]

[13] Guardian Digital (2005) Windows vs. Linux in Corporate Environments: The Advantages of Windows Available at: http://www.guardiandigital.com/pfiles/GuardianDigital_Linux_vs_Windows.pdf [Accessed 20 February 2018]

[14] Cybersource ${ }^{\circledR}$ (2004) Linux VS Windows Total Cost of Ownership Comparison. Available at: https://static.lwn.net/images/pdf/cybersource-tco-study.pdf [Accessed 21 February 2018]

[15] Carson, A. (2011) What Are the Advantages and Disadvantages of Mac OS?: Advantages of Mac OS. Available at: http://www.brighthub.com/computing/mac-platform/articles/73326.aspx [Accessed 9 March 2018]

[16] Hayat, S. (2013) Scl 3: Operating System and File Management. Available at: http://prxhayat2group10.blogspot.com/ [Accessed 7 March 2018]

[17] Informatics-tech (2013) Linux vs Macintosh vs Windows (unbiased comparison) Available at: http://www.informatics-tech.com/linux-vs-mac-vs-windows-unbiased-comparison.htmlf[Accessed 21 February 2018]

[18] Tutorialspoint [2016] Learning Operating System - Linux [online] Available at: http://www.tutorialspoint.com/operating_system/os_linux.htm [Accessed 10 March 2018] 